\title{
Resting-state networks do not determine cognitive function networks: a commentary on Campbell and Schacter (2016)
}

\section{Simon W. Davis, Matthew L. Stanley, Morris Moscovitch \& Roberto Cabeza}

To cite this article: Simon W. Davis, Matthew L. Stanley, Morris Moscovitch \& Roberto Cabeza (2016): Resting-state networks do not determine cognitive function networks: a commentary on Campbell and Schacter (2016), Language, Cognition and Neuroscience, DOI: 10.1080/23273798.2016.1252847

To link to this article: http://dx.doi.org/10.1080/23273798.2016.1252847

曲 Published online: 06 Nov 2016.

Submit your article to this journal $\pi$

Џ Article views: 227

View related articles $\sqsubset \pi$

View Crossmark data ¿ 


\title{
Resting-state networks do not determine cognitive function networks: a commentary on Campbell and Schacter (2016)
}

\author{
Simon W. Davis $\mathbb{C l}^{\mathrm{a}}$, Matthew L. Stanley ${ }^{\mathrm{b}}$, Morris Moscovitch ${ }^{\mathrm{c}}$ and Roberto Cabeza ${ }^{\mathrm{b}}$ \\ ${ }^{a}$ Neurology, Duke University School of Medicine, Durham, NC, USA; ${ }^{b}$ Center for Cognitive Neuroscience, Duke Institute for Brain Sciences, Duke

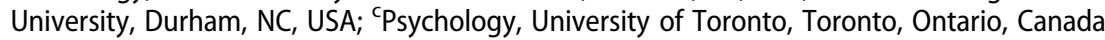

ARTICLE HISTORY Received 12 October 2016; Accepted 19 October 2016

In their insightful article, Campbell and Schacter identify several potential problems with the interpretation of resting-state functional magnetic resonance imaging ( $\mathrm{fMRI}$ ) data in the context of cognitive neuroscience of ageing, and they argue that resting-state studies must be complemented by cognitively based task-related studies if we are to achieve a proper understanding of ageing phenomena at the systems level. They discuss, for example, the practice of relating resting-state network (RSN) properties to cognitive measures and treating RSN measures as a trait. We agree with the issues they have raised and some of the solutions they have proposed, but we believe the problems with RSN data interpretation extend beyond the ageing domain, affecting the whole field of cognitive neuroscience. The goal of cognitive neuroscience is to understand the neural mechanisms of cognition, describe patterns of interactions that support cognitive processes, and make useful and meaningful predictions about which brain states facilitate different cognitions. Achieving these goals requires understanding the brain networks that are activated during cognitive tasks and support successful cognitive performance, which we call here cognitive function networks (CFNs). The now widespread use of RSNs in cognitive neuroscience is often based on the assumption that RSNs determine, modulate, or even comprise CFNs. There are, however, at least four problems with this commonly held assumption, and with the RSN approach to explaining cognition.

RSNs are spatially and topologically different from CFNs, and as Campbell and Schacter are keen to point out a central problem with the use of RSNs in cognitive neuroscience is that they do not shed light on the neural instantiation of cognitive processes. Although active regions in different CFNs often partially overlap with RSNs (Power, Schlaggar, Lessov-Schlaggar, \& Petersen, 2013), there is now abundant, direct evidence that patterns of co-activation, or connectivity, of RSNs are significantly and meaningfully different than those of the CFNs recruited by a variety of cognitive tasks, including working memory (Rzucidlo, Roseman, Laurienti, \& Dagenbach, 2013; Stanley et al., 2015) semantic decision-making (DeSalvo, Douw, Takaya, Liu, \& Stufflebeam, 2014), multisensory stimulation (Moussa et al., 2011), executive functioning (Bolt, Laurienti, Lyday, Morgan, \& Dagenbach, 2016), and auditory detection (Sadaghiani, Poline, Kleinschmidt, \& D'Esposito, 2015). Such differences are evident even when the tasks are not event-related but made to resemble resting-state tasks (Geerligs, Renken, Saliasi, Maurits, \& Lorist, 2015), even to the point of using comparable paradigms (selfdirected, uninterrupted thought of equal duration; see Bellana, Lui, Diamond, Grady, \& Moscovitch, 2016).

We can observe these topological breakdowns not just in gross differences from rest to task, but often as a function of cognitive performance. At the regional level, for example, Leech, Kamourieh, Beckmann, and Sharp (2011) found that as task difficulty increases, traditional posterior cingulate cortex (PCC) parcellations fractionate, and they show reduced integration within the default mode network (DMN) at rest and less anti-correlation with the more dorsal cognitive control network activated by the task. At the level of large-scale networks, Stanley, Dagenbach, Lyday, Burdette, and Laurienti (2014) found that the modular organisation of default mode regions became significantly less consistent across participants with increasing working memory load, whereas the modular organisation of regions traditionally considered to support working memory processes became more consistent with increasing working memory load. Thus, standard resting-state assignments become rather meaningless when considered within the context of parametric modulations in a cognitive task. These differences in network 
organisation between RSNs and CFNs have been observed across multiple spatial and temporal scales and using diverse methods of network construction. Thus, it is unclear to what extent knowledge of RSNs helps us understand CFNs. We review four specific problems related to the use of RSNs in cognitive neuroscience, in the context of both regional changes and large-scale network-level analyses.

First, regions belonging to the same RSN are often dissociated in different CFNs (dissociation problem). It has been assumed that regions co-activated during rest also tend to be coactivated during cognitive tasks, but this is often not the case. The RSN known as DMN (Gusnard, Raichle, \& Raichle, 2001), for example, traditionally consists of anterior cingulate cortex (ACC) and PCC, ventromedial prefrontal cortex (vmPFC), dorsomedial prefrontal cortex (dmPFC), inferior parietal lobule, lateral temporal cortex, and the hippocampus. Almost every possible paired combination of these regions has been dissociated in the cognitive neuroimaging literature. For instance, vmPFC is activated when processing information about the self and dmPFC with information about others (Mitchell, Macrae, \& Banaji, 2006; St Jacques, Conway, Lowder, \& Cabeza, 2011). PCC is coactivated with the hippocampus during episodic retrieval but not during episodic encoding (Huijbers, Pennartz, Cabeza, \& Daselaar, 2011). The hippocampus is activated during the encoding of both semantically related vs. semantically unrelated word pairs, whereas lateral temporal cortex is activated only for semantically related pairs (Prince, Tsukiura, \& Cabeza, 2007). It has been argued, however, that the DMN is composed of several subsystems and that only ACC and PCC form the true "core" of this network (Andrews-Hanna, Reidler, Sepulcre, Poulin, \& Buckner, 2010). Yet, ACC and PCC are also dissociated during cognitive tasks (Sestieri, Corbetta, Romani, \& Shulman, 2011).

Citing Geerligs et al. (2015), Campbell and Schacter note that such dissociations occur across task states even when the tasks (rest, watching movies, and lowlevel audio-visual) are not event-related, though some overlap does exist. Critically, they note, ageing effects differed across tasks. Similar dissociations were obtained by Bellana et al. (in press) even when two task-related paradigms - retrieving past memories and imagining future events - were equated with resting-state paradigms by having each block consist of self-directed, uninterrupted thought of equal duration. The similarity in functional connectivity between the DMN and two task-related activities accounted for only $40-50 \%$ of the variance. The medial temporal regions of the DMN were preferentially coupled with one another during episodic retrieval and future imagining, whereas the non-medial temporal regions of the DMN (e.g. medial prefrontal cortex, lateral temporal cortex, and temporal pole) were preferentially coupled during rest (Bellana et al., in press). The effects of ageing on these task-related connectivity patterns have yet to be tested. Dissociations among components are not specific for the DMN and can be found for most RSNs. Thus, knowing that two brain regions "belong" to the same RSN does not inform whether they will be coactivated and collaborate during a cognitive task, challenging the usefulness of RSN data for cognitive neuroscience.

Second, regions belonging to different RSNs often become associated in CFNs (association problem). This is the reverse of the previous problem: regions disconnected during rest become connected during cognitive task. For example, the hippocampus is typically classified as a component of the DMN, whereas left ventrolateral PFC has been classified as a component of the language network (Lee, Smyser, \& Shimony, 2013). Yet, the hippocampus and left ventrolateral PFC are frequently coactivated during episodic memory encoding (Kim, 2011, 2014; Spaniol et al., 2009). Moreover, functional connectivity between these regions is associated with successful episodic encoding (Schott et al., 2013; Wing, Marsh, \& Cabeza, 2013), indicating that their collaboration is important for this task. Additionally, the bilateral insula and the ACC are often regarded as the core of the "salience network", though the number of studies relating resting connectivity in these regions to cognitive markers of awareness is limited (though, see Deen, Pitskel, \& Pelphrey, 2011; Taylor, Seminowicz, \& Davis, 2009); perhaps more prevalent in the literature are connectivity results between the insula and the amygdala during emotion regulation (Townsend et al., 2013), left insula and anterior PFC connectivity underlying awareness (Craig, 2009), or sensorimotor representations relying on insular connectivity with posterior PFC/parietal regions (Riecker et al., 2005). Thus, the association of a particular set of regions during rest often obscures more specific functional associations observed during a task.

Third, the same brain region can be part of multiple CFNs and have different connectivity in each of them (versatility problem). An implicit assumption is that the connectivity of a brain region is relatively stable. Yet, CFN evidence shows that the same brain region can (1) contribute to many different cognitive processes and (2) have a different pattern of connectivity for each of them. For example, the same regions ventral parietal cortex (VPC) may contribute to episodic memory retrieval, perceptual reorienting, number processing, language comprehension, and theory of mind (Cabeza, Ciaramelli, \& Moscovitch, 2012a, 2012b). Although the 
same region is involved in different tasks, its pattern of functional connectivity changes. For example, VPC interacts with occipital cortex during visual perceptual orienting but with the medial temporal lobe during episodic memory retrieval. In other words, brain regions are versatile; they can form part of multiple CFNs and collaborate with different regions depending on the process they mediate. To emphasise that collaborations among brain regions are process-specific and transitory, we introduced the term process-specific alliances (PSAs) (Cabeza \& Moscovitch, 2013; Moscovitch, Cabeza, Winocur, \& Nadel, 2016). A PSA is a small "team" of brain regions that is rapidly assembled to mediate a cognitive process and quickly disassembled when no longer needed. A cognitive task involves the operation of multiple PSAs. During episodic memory retrieval, for instance, a PSA between left ventrolateral PFC and left temporal cortex may mediate semantic processing of the retrieval cue; a PSA between left dorsolateral PFC and dorsal parietal cortex, the control of the memory search; a PSA between the hippocampus and posterior sensory cortices, the reactivation of stored memory traces; and a PSA including these regions plus VPC, the subjective experience of remembering (Moscovitch et al., 2016). Although these seven regions could be described as the episodic memory retrieval CFN, each of these regions forms a part of other CFNs (e.g. VPC during theory of mind). RSN research cannot inform us about versatility of brain regions and their rapidly changing connectivity patterns.

Campbell and Schacter draw similar conclusions from studies showing dynamic configuration of networks over time or from task to rest. Although some hints as to these changes can be gleaned from event-related fMRI studies, appreciating them fully will require developing techniques, such as magnetoencephalography and intracranial recordings, which have both the structural and temporal specificity needed to identify the task-related PSAs. Once the necessary techniques are developed, such studies may become invaluable in tracking agerelated cognitive changes which may be related as much to the ability to shift from one PSA, or network, to another as to the PSAs and networks that are formed.

Finally, the role of a region within a network cannot be inferred from its role during rest because it varies depending on performance (performance-specificity problem). In other words, regions that may appear as unimportant in an RSN may be critically important in a similar CFN, and vice versa. For example, although many DMN regions are activated during episodic memory retrieval, DMN components that appear as very important during rest can be secondary during episodic retrieval. As noted before, ACC and the PCC are considered the most important, "core" regions of the DMN (AndrewsHanna et al., 2010). Yet, these two regions are often inactive during episodic retrieval, particularly the ACC (Rugg \& Vilberg, 2013). Conversely, the hippocampus is not topologically central within the DMN (Hagmann et al., 2008; Tomasi \& Volkow, 2010; van den Heuvel, Kahn, Goni, \& Sporns, 2012), but it is arguably the most important brain region for supporting episodic memory processes. To identify regions that are topologically central for a CFN, it is critical to link topological properties of networks to cognitive performance. In Geib, Stanley, Wing, Laurienti, and Cabeza (2015), for example, participants recalled visual scenes and rated the vividness of those memories in the scanner. Separate task-based networks were constructed for vivid and dim memories. When centrality analyses were separately performed on these distinct networks, the hippocampal nodes (right and left) were ranked among the least central nodes in the network, with a ranking of 67th and 68th in the vivid retrieval network and 85th and 89th in the dim retrieval network. Yet, when centrality analyses were based on the difference between vivid and dim networks, thereby isolating network differences related to retrieval success, the hippocampus was ranked 1st in the right hemisphere and 2 nd in the left hemisphere (Geib et al., 2015). Thus, it is not possible to infer the topological importance of a node within a CFN on the basis of RSN data alone. In discussing the findings of Salami, Pudas, and Nyberg (2014), Campbell and Schacter note a related problem with respect to changes in connectivity of the same region at rest and during task performance. Age-related increases in hippocampal connectivity at rest are associated with decreases during encoding, and both are predictive of poorer subsequent memory in ageing.

In conclusion, there are at least four problems with the assumption that RSNs determine or constrain CFNs: (1) regions connected within an RSN become disconnected in a CFN, (2) regions disconnected in an RSN become connected in a CFN, (3) a particular region within an RSN may have multiple roles in distinct CFNs, and (4) the role a region within a CFN may depend upon performance and cannot be inferred from RSN data alone. Thus, to understand CFNs, it is better, in our view, to focus on task-related functional connectivity data and investigate changes in topological properties between cognitive tasks or conditions at both local and global levels. We believe the potential contributions of RSNs to understanding CFNs, which are the networks that cognitive neuroscience really wants to understand, have been exaggerated. RSNs and CFNs are different; regions that go together in an RSN are often separate in CFNs; and the role of a region in a CFN cannot be predicted from its activity or connectivity patterns during rest. 
These network-level considerations have important implications for ageing studies, as RSNs based on large lifespan samples are increasingly treated as "benchmark" parcellations (Glasser et al., 2016; Van Essen et al., 2013), and used to identify any number of salient age-related changes in brain network function (Betzel, Byrge, He, Goni, Zuo, \& Sporns, 2014). We agree with Morcom and Fletcher that the "resting state" constitutes another "task state" (Morcom \& Fletcher, 2007), one in which participants may report any number of explicit mental activities (Fox, Spreng, Ellamil, Andrews-Hanna, \& Christoff, 2015), despite the technician's advisement to "stare at the cross and try not to think of anything". The evidence reviewed above clearly shows that what is functionally connected during rest need not be functionally connected during certain tasks. Resting-state data remain useful insofar as they help to represent "a point of comparison across studies, which then require further testing with a task-based approach" (p. 10). It is clear, however, from Campbell and Schacter's commentary that a number of issues cloud the interpretation of the relationship between age-related cognitive changes and the resting state. There is no free lunch in the elucidation of functional role of distinguishable brain networks, and we believe the use of task-related and naturalistic study paradigms will help to clarify the conditions that identify robust and reproducible neurocognitive networks.

\section{Disclosure statement}

No potential conflict of interest was reported by the authors.

\section{Funding}

S. W. D., M. L. S., and R. C. are supported by the National Institute on Aging [NIA R01AG19731] to R. C. M. M. is supported by Canadian Institute of Health Research (CIHR) grant [MGP6694].

\section{ORCID}

Simon W. Davis (D) http://orcid.org/0000-0002-5943-0756

\section{References}

Andrews-Hanna, J. R., Reidler, J. S., Sepulcre, J., Poulin, R., \& Buckner, R. L. (2010). Functional-anatomic fractionation of the brain's default network. Neuron, 65(4), 550-562. doi:10. 1016/j.neuron.2010.02.005

Bellana, B., Liu, Z. X., Diamond, N. B., Grady, C. L., \& Moscovitch, M. (2016). Similarities and differences in the default mode network across rest, retrieval, and future imagining. Human Brain Mapping. doi:10.1002/hbm.23445
Betzel, R. F., Byrge, L., He, Y., Goni, J., Zuo, X. N., \& Sporns, O. (2014). Changes in structural and functional connectivity among resting-state networks across the human lifespan. Neuroimage, 102(Pt 2), 345-357. doi:10.1016/j.neuroimage. 2014.07.067

Bolt, T., Laurienti, P. J., Lyday, R., Morgan, A., \& Dagenbach, D. (2016). Graph-theoretical study of functional changes associated with the lowa gambling task. Frontiers in Human Neuroscience, 10, 314. doi:10.3389/fnhum.2016.00314

Cabeza, R., Ciaramelli, E., \& Moscovitch, M. (2012a). Cognitive contributions of the ventral parietal cortex: An integrative theoretical account. Trends in Cognitive Sciences, 16, 338352.

Cabeza, R., Ciaramelli, E., \& Moscovitch, M. (2012b). Response to Nelson et al.: Ventral parietal subdivisions are not incompatible with an overarching function. Trends in Cognitive Sciences, 16(8), 400-401. doi:10.1016/j.tics.2012.06.015

Cabeza, R., \& Moscovitch, M. (2013). Memory systems, processing modes, and components: Functional neuroimaging evidence. Perspectives on Psychological Science, 8(1), 49-55. doi:10.1177/1745691612469033

Craig, A. D. (2009). How do you feel - now? The anterior insula and human awareness. Nature Reviews Neuroscience, 10(1), 59-70. doi:10.1038/nrn2555

Deen, B., Pitskel, N. B., \& Pelphrey, K. A. (2011). Three systems of insular functional connectivity identified with cluster analysis. Cerebral Cortex, 21(7), 1498-1506. doi:10.1093/cercor/ bhq186

DeSalvo, M. N., Douw, L., Takaya, S., Liu, H., \& Stufflebeam, S. M. (2014). Task-dependent reorganization of functional connectivity networks during visual semantic decision making. Brain and Behavior, 4(6), 877-885. doi:10.1002/brb3.286

Fox, K. C., Spreng, R. N., Ellamil, M., Andrews-Hanna, J. R., \& Christoff, K. (2015). The wandering brain: Meta-analysis of functional neuroimaging studies of mind-wandering and related spontaneous thought processes. Neuroimage, 111, 611-621. doi:10.1016/j.neuroimage.2015.02.039

Geerligs, L., Renken, R. J., Saliasi, E., Maurits, N. M., \& Lorist, M. M. (2015). A brain-wide study of age-related changes in functional connectivity. Cerebral Cortex, 25(7), 1987-1999. doi:10.1093/cercor/bhu012

Geib, B. R., Stanley, M. L., Wing, E. A., Laurienti, P. J., \& Cabeza, R. (2015). Hippocampal contributions to the large-scale episodic memory network predict vivid visual memories. Cerebral Cortex. doi:10.1093/cercor/bhv272

Glasser, M. F., Coalson, T. S., Robinson, E. C., Hacker, C. D., Harwell, J., Yacoub, E., ... Van Essen, D. C. (2016). A multimodal parcellation of human cerebral cortex. Nature, 536 (7615), 171-178. doi:10.1038/nature18933

Gusnard, D. A., Raichle, M. E., \& Raichle, M. E. (2001). Searching for a baseline: Functional imaging and the resting human brain. Nature Reviews Neuroscience, 2(10), 685-694. doi:10. 1038/35094500

Hagmann, P., Cammoun, L., Gigandet, X., Meuli, R., Honey, C. J., Wedeen, V. J., \& Sporns, O. (2008). Mapping the structural core of human cerebral cortex. PLoS Biology, 6(7), e159. doi:10.1371/journal.pbio.0060159

van den Heuvel, M. P., Kahn, R. S., Goni, J., \& Sporns, O. (2012). High-cost, high-capacity backbone for global brain communication. Proceedings of the National Academy of Sciences of the United States of America, 109(28), 1137211377. doi:10.1073/pnas.1203593109 
Huijbers, W., Pennartz, C. M. A., Cabeza, R., \& Daselaar, S. M. (2011). The hippocampus is coupled with the default network during memory retrieval but not during memory encoding. Plos One, 6(4), e17463. doi:e1746310.1371/ journal.pone.0017463

Kim, H. (2011). Neural activity that predicts subsequent memory and forgetting: A meta-analysis of $74 \mathrm{fMRl}$ studies. Neuroimage, 54(3), 2446-2461. doi:10.1016/j.neuroimage. 2010.09.045

Kim, H. (2014). Encoding and retrieval along the long axis of the hippocampus and their relationships with dorsal attention and default mode networks: The HERNET model. Hippocampus. doi:10.1002/hipo.22387

Lee, M. H., Smyser, C. D., \& Shimony, J. S. (2013). Resting-state fMRI: A review of methods and clinical applications. American Journal of Neuroradiology, 34(10), 1866-1872. doi:10.3174/ajnr.A3263

Leech, R., Kamourieh, S., Beckmann, C. F., \& Sharp, D. J. (2011). Fractionating the default mode network: Distinct contributions of the ventral and dorsal posterior cingulate cortex to cognitive control. Journal of Neuroscience, 31(9), 32173224. doi:10.1523/JNEUROSCI.5626-10.2011

Mitchell, J. P., Macrae, C. N., \& Banaji, M. R. (2006). Dissociable medial prefrontal contributions to judgments of similar and dissimilar others. Neuron, 50(4), 655-663.

Morcom, A. M., \& Fletcher, P. C. (2007). Does the brain have a baseline? Why we should be resisting a rest. Neuroimage, 37(4), 1073-1082. doi:10.1016/j.neuroimage.2006.09.013

Moscovitch, M., Cabeza, R., Winocur, G., \& Nadel, L. (2016). Episodic memory and beyond: The hippocampus and neocortex in transformation. In S. T. Fiske (Ed.), Annual review of psychology (Vol. 67, pp. 105-134). Palo Alto, CA: Annual Reviews.

Moussa, M. N., Vechlekar, C. D., Burdette, J. H., Steen, M. R., Hugenschmidt, C. E., \& Laurienti, P. J. (2011). Changes in cognitive state alter human functional brain networks. Frontiers in Human Neuroscience, 5, 83. doi:10.3389/fnhum.2011.00083

Power, J. D., Schlaggar, B. L., Lessov-Schlaggar, C. N., \& Petersen, S. E. (2013). Evidence for hubs in human functional brain networks. Neuron, 79(4), 798-813. doi:10.1016/j.neuron.2013.07. 035

Prince, S. E., Tsukiura, T., \& Cabeza, R. (2007). Distinguishing the neural correlates of episodic memory encoding and semantic memory retrieval. Psychological Science, 18(2), 144-151. doi:10.1111/j.1467-9280.2007.01864.x

Riecker, A., Mathiak, K., Wildgruber, D., Erb, M., Hertrich, I., Grodd, W., \& Ackermann, H. (2005). fMRI reveals two distinct cerebral networks subserving speech motor control. Neurology, 64(4), 700-706. doi:10.1212/01.WNL.0000152156. 90779.89

Rugg, M. D., \& Vilberg, K. L. (2013). Brain networks underlying episodic memory retrieval. Current Opinion in Neurobiology, 23(2), 255-260. doi:10.1016/j.conb.2012.11.005

Rzucidlo, J. K., Roseman, P. L., Laurienti, P. J., \& Dagenbach, D. (2013). Stability of whole brain and regional network topology within and between resting and cognitive states. PLoS One, 8(8), e70275. doi:10.1371/journal.pone.0070275

Sadaghiani, S., Poline, J. B., Kleinschmidt, A., \& D’Esposito, M. (2015). Ongoing dynamics in large-scale functional connectivity predict perception. Proceedings of the National
Academy of Sciences of the United States of America, 112 (27), 8463-8468. doi:10.1073/pnas.1420687112

Salami, A., Pudas, S., \& Nyberg, L. (2014). Elevated hippocampal resting-state connectivity underlies deficient neurocognitive function in aging. Proceedings of the National Academy of Sciences of the United States of America, 111(49), 1765417659. doi:10.1073/pnas.1410233111

Schott, B. H., Wustenberg, T., Wimber, M., Fenker, D. B., Zierhut, K. C., Seidenbecher, C. I., ... Richardson-Klavehn, A. (2013). The relationship between level of processing and hippocampal-cortical functional connectivity during episodic memory formation in humans. Human Brain Mapping, 34(2), 407-424. doi:10.1002/hbm.21435

Sestieri, C., Corbetta, M., Romani, G. L., \& Shulman, G. L. (2011). Episodic memory retrieval, parietal cortex, and the default mode network: Functional and topographic analyses. Journal of Neuroscience, 31(12), 4407-4420. doi:10.1523/ jneurosci.3335-10.2011

Spaniol, J., Davidson, P. S. R., Kim, A. S. N., Han, H., Moscovitch, M., \& Grady, C. L. (2009). Event-related fMRI studies of episodic encoding and retrieval: Meta-analyses using activation likelihood estimation. Neuropsychologia, 47(8-9), 17651779. doi:10.1016/j.neuropsychologia.2009.02.028

St Jacques, P. L., Conway, M. A., Lowder, M. W., \& Cabeza, R. (2011). Watching my mind unfold versus yours: An fMRI study using a novel camera technology to examine neural differences in self-projection of self versus other perspectives. Journal of Cognitive Neuroscience, 23(6), 1275-1284. doi:10.1162/jocn.2010.21518

Stanley, M. L., Dagenbach, D., Lyday, R. G., Burdette, J. H., \& Laurienti, P. J. (2014). Changes in global and regional modularity associated with increasing working memory load. Frontiers in Human Neuroscience, 8, 3889. doi:10.3389/ fnhum.2014.00954

Stanley, M. L., Simpson, S. L., Dagenbach, D., Lyday, R. G., Burdette, J. H., \& Laurienti, P. J. (2015). Changes in brain network efficiency and working memory performance in aging. PLoS One, 10(4), e0123950. doi:10.1371/journal.pone. 0123950

Taylor, K. S., Seminowicz, D. A., \& Davis, K. D. (2009). Two systems of resting state connectivity between the insula and cingulate cortex. Human Brain Mapping, 30(9), 27312745. doi:10.1002/hbm.20705

Tomasi, D., \& Volkow, N. D. (2010). Functional connectivity density mapping. Proceedings of the National Academy of Sciences of the United States of America, 107(21), 98859890. doi:10.1073/pnas.1001414107

Townsend, J. D., Torrisi, S. J., Lieberman, M. D., Sugar, C. A., Bookheimer, S. Y., \& Altshuler, L. L. (2013). Frontal-amygdala connectivity alterations during emotion downregulation in bipolar I disorder. Biological Psychiatry, 73(2), 127-135. doi:10.1016/j.biopsych.2012.06.030

Van Essen, D. C., Smith, S. M., Barch, D. M., Behrens, T. E., Yacoub, E., Ugurbil, K., \& WU-Minn HCP Consortium (2013). The WU-Minn Human Connectome Project: An overview. Neuroimage, 80, 6279. doi:10.1016/j.neuroimage.2013.05.041

Wing, E. A., Marsh, E. J., \& Cabeza, R. (2013). Neural correlates of retrieval-based memory enhancement: An fMRI study of the testing effect. Neuropsychologia, 51(12), 2360-2370. doi:10. 1016/j.neuropsychologia.2013.04.004 